\title{
Schnelle Diagnose der septischen Arthritis
}

\author{
Berthoud O et al. Performance of a new rapid \\ diagnostic test the lactate/glucose ratio of \\ synovial fluid for the diagnosis of septic arthritis. \\ Joint Bone Spine 2020; 87: 343-350. doi: \\ 10.1016/j.jbspin.2020.03.009
}

Bei septischer Arthritis kann die Abgrenzung von anderen Formen der akuten entzündlichen Arthritis schwierig sein. Bisher dienen zeitaufwendige Synovialflüssigkeitskulturen zur Identifizierung eines Erregers. Der Nachweis einer bakteriellen Infektion könnte jedoch schneller durch Bestimmung des bakteriellen Glukoseverbrauchs bzw. der Laktatproduktion erfolgen. Diese Arbeit untersucht, ob die Messung dieser Parameter im Synovium als Biomarker zur Diagnose der septischen Arthritis geeignet ist.

\section{Methoden}

Die monozentrische Querschnittsstudie umfasste 233 Patienten (Durchschnittsalter 61,8 Jahre, 64,8\% Männer) mit akutem Gelenkerguss (<30 Tage). Bei allen Patienten wurde prospektiv Synovialflüssigkeit am nativen Gelenk gewonnen und Synovialflüssigkeitskulturen angelegt. $20 \%$ der Patienten erhielten eine Antibiotikatherapie vor der Punktion. Eine septische Arthritis wurde anhand der Newman'schen Kriterien definiert. Zur Beurteilung der diagnostischen Leistung des Lactat/Glucose-Tests wurden Receiver Operating Characteristic (ROC)-Kurven mit Area under the curve (AUC), Sensitivitäten (Se), Spezifitäten (Sp), LR + und LR- berechnet. Zur Kontrolle wurden Synovialflüssigkeitskulturen mit GramFärbung, Kristallanalysen, Zählungen der Leukozyten im Synovium, Laktat- und Glukose-Assays durchgeführt.

\section{Ergebnisse}

Von den 233 untersuchten Patienten hatten 25 (10,7\%) eine septische Arthritis und 208 eine nicht-septische Arthritis (104 kristallin-induzierte Arthritis, 15 rheumatoide Arthritis, 8 Spondyloarthritis, 6 reaktive Arthritis und 75 akute Arthritis undifferenzierten Ursprungs). In der septischen Gruppe 
waren die Laktatspiegel höher und die AUC von Laktat betrug 0,795. Die Glukosespiegel waren niedriger und die AUC von Glukose betrug 0,833. Das synoviale Laktat/Glucose-Verhältnis diskriminierte besser als die einzelnen synovialen Laktat- bzw. Glukose-Tests (AUC: 0,859 [0,772-0,945]). Der beste Schwellenwert für das synoviale Laktat/Glukose-Verhältnis zur Unterscheidung zwischen septischer und nicht septischer Arthritis lag bei 5 Se $52 \%(0,34-0,7)$, Sp $98,1 \%(0,95-0,99), \mathrm{LR}+27,0(9,50-76,00)$. Septische Arthritis in kleinen Gelenken mit einem geringen Volumen an Synovialflüssigkeit wurde nicht einbezogen.

\section{FAZIT}

Insbesondere anhand des synovialen Laktat/Glukose-Verhältnisses lässt sich die septische Arthritis effektiv und sehr schnell von anderen Typen akuter Arthritis unterscheiden. Die Stichprobe war allerdings zu klein, um einen möglichen Unterschied zwischen Laktat- und Glukosespiegel mit oder ohne Antibiotikatherapie vor der Punktion zu zeigen. Eine mögliche Einschränkung war der geringere Anteil an septischer Arthritis (10,7\%) im Vergleich zu den üblichen $15 \%$.

Dr. Markus Numberger, Kandel 